INPLASY

PROTOCOL

To cite: Hu et al. Nonpharmacological Intervention for Rehabilitation of Post-

Stroke Spasticity: a Protocol for Systematic review and Network meta-analysis. Inplasy protocol 202140059. doi:

10.37766/inplasy2021.4.0059

Received: 10 April 2021

Published: 10 April 2021

Corresponding author:

Deyu Cong

cong_de_yu@sina.com

Author Affiliation:

Changchun University of

Chinese Medicine

Support: National Key R\&D

Program of CN.

Review Stage at time of this submission: Preliminary

searches.

Conflicts of interest:

None declared.

\section{Non-pharmacological Intervention for Rehabilitation of Post-Stroke Spasticity: a Protocol for Systematic review and Network meta-analysis}

\author{
Hu, G1; Zhang, H²; Wang, Y3; Cong, D4.
}

Review question / Objective: We will conduct a network meta analysis of different types of non-pharmacological RCT studies on post-stroke spasticity(PSS) to find out the differences in the efficacy of different types of nonpharmacological therapies on PSS.

Information sources: The following databases will be searched electronically, including four English literature databases (i.e., PubMed, Embase, MEDLINE, and Cochrane Library) and two Chinese literature databases (i.e., China National Knowledge Infrastructure and VIP). In addition, we will retrieve unpublished protocols and summarize the results by searching the clinical trial registry at https:// ClinicalTrials.gov. We will also manually retrieve relevant conference reports and contact experts in the field and corresponding authors to obtain important information that cannot be obtained by the above retrieval.

INPLASY registration number: This protocol was registered with the International Platform of Registered Systematic Review and Meta-Analysis Protocols (INPLASY) on 10 April 2021 and was last updated on 10 April 2021 (registration number INPLASY202140059).

\section{INTRODUCTION}

Review question / Objective: We will conduct a network meta analysis of different types of non-pharmacological RCT studies on post-stroke spasticity(PSS) to find out the differences in the efficacy of different types of non-pharmacological therapies on PSS.
Rationale: Network meta analysis enables the comparison of multiple interventions to incorporate clinical evidence for direct and indirect treatment comparisons in treatment and related trial networks. And the Bayesian NMA method was adopt in the present work to find out the difference of therapeutic effects of PSS between 
different types of non-pharmacological interventions.

Condition being studied: Stroke is characterized by high morbidity, mortality, and disability. Post-stroke spasticity (PSS) is the most common complication of stroke. It is estimated that approximately $20 \%-40 \%$ of stroke survivors will have limb spasms. Research on the occurrence time and degree of PSS showed that the incidence of spasm within 1 month after stroke was $42.6 \%$, of which severe spasm was $15.6 \%$. The incidence of spasms at 3 months is approximately $19 \%$. The incidence of spasms within 6 months in stroke patients is $21.7 \% \sim 23 \%$.PSS seriously affects the motor function of patients, and therefore seriously affects the living standards and prognosis of patients. Therefore, it is very important to select a safe and cost-effective PSS treatment and rehabilitation method. Treatments of PSS include oral anti-spastic medication, BTX-A injections, surgical interventions, and physiotherapy or a combination of the aforementioned therapies. A study has shown that medication can relieve spasms caused by central nerve injury and may also cause muscle weakness.In clinical treatments, physiotherapy is a rehabilitation intervention for PSS, such as Bobath, which is widely used in the treatment of PSS. To improve the rehabilitation efficiency of PSS, most medical institutions combine a variety of characteristic non-pharmacological therapies based on conventional rehabilitation therapies for PSS. Hospitals of traditional Chinese medicine (or rehabilitation departments of traditional Chinese medicine) often combine electric acupuncture, Tui-na, medicinal bath, and other therapies in the rehabilitation of PSS.It is difficult to optimize the clinical rehabilitation program of PSS because of the wide variety of non-pharmacological therapies that have rehabilitative effects on PSS. Therefore, it is of great significance for the rehabilitation of PSS to select intervention methods with higher cost performance among various interventions. To date, this is the first time that network meta-analysis has been used to compare currently available methods for multiple interventions to determine the effectiveness of non-pharmacological interventions in the PSS.

\section{METHODS}

Search strategy: We will search for RCTs on non-pharmacological interventions for post-stroke spasticity, and the search time limit is from its establishment to April 2021. The search used a combination of subject words and free words, and the search strategy was determined after multiple presearches. The search terms included post-stroke, after stroke, spastic hemiplegia, spasticity, limb spasm, treatment, intervention, management, rehabilitation, RCT and randomization. Meanwhile, we will search the literature included in the research reference and original literature, which are subject related and included in systematic reviews, to supplement and obtain relevant literature and ensure the recall ratio.

Participant or population: The cases included in the trial are all patients with post-stroke spasticity (as diagnosed using any recognised diagnostic criteria), not limited by age and race.

Intervention: The treatment group adopted non-pharmacological intervention (ie, acupuncture, dry acupuncture, tuina and medicated bath and music therapy, etc., without restricting the choice of operation method and course of treatment).

Comparator: The control group adopted internationally recognized treatment methods or routine treatment(such as rehabilitation training).

Study designs to be included: All clinical randomized controlled trials (RCTs) of nonpharmacological therapy for PSS will be included in the review.

Eligibility criteria: All clinical randomized controlled trials (RCTs) of nonpharmacological therapy for PSS will be included in the review. 
Information sources: The following databases will be searched electronically, including four English literature databases (i.e., PubMed, Embase, MEDLINE, and Cochrane Library) and two Chinese literature databases (i.e., China National Knowledge Infrastructure and VIP). In addition, we will retrieve unpublished protocols and summarize the results by searching the clinical trial registry at https://ClinicalTrials.gov. We will also manually retrieve relevant conference reports and contact experts in the field and corresponding authors to obtain important information that cannot be obtained by the above retrieval.

Main outcome(s): The primary outcome indicator was the assessment of motor function, and the included RCTs included at least one of the Modified Ashworth Scale(MAS)and the Fugl-Meyer Assessment (FMA).

Additional outcome(s): Secondary outcome indicators were daily living ability assessment, including the Barthel index (BI) rating scale and daily living ability scale (ADL).

Data management: Two independent reviewers extracted data from selected studies using pilot-tested data forms. They will include the following information: author, year of publication, study population, study design, number of patients randomized and treated, number of patients analyzed, baseline analysis, random sequence generation, allocation concealment method, blinding method, imputation method, withdrawals of data, interventions, controls, medication records, and primary and secondary outcomes at all reported time points. To investigate the characteristics of non-pharmacological intervention effects, we extracted data on age, sex, population, number and duration of treatment sessions, features of nonpharmacological intervention (such as frequency of stimulation and point of treatment), features of control interventions (sham methods or standard treatment details), and patient expectations. We also document for each outcome of the percentage of missing values reported in the study, and any disagreement on data collection will be resolved through discussions or negotiations with the third arbitrator. If the data provided in the study are unclear, missing, or presented in a form that is not extractable or difficult to extract reliably, we will contact the author of the study for clarification.

Quality assessment / Risk of bias analysis: According to the Cochrane Collaboration's tool for assessing risk of bias provided by the Cochrane Handbook for Systematic Reviews of Interventions, seven dimensions will be assessed from 7 dimensions: random sequence generation, allocation concealment, blinding of patients, blinding of testers, blinding of outcome evaluators, outcome data incompletion, and selective reporting of seven dimensions for evaluation. The results of the assessment were divided into three levels: low risk, unclear, and high risk. The assessment will be conducted independently by two trained research members, and the inconsistencies will be resolved through intragroup discussions, contacting authors to determine details with the third-party arbitrator.

Strategy of data synthesis: The synthesis will be performed by generating a forest plot for meta-regression. This plot does not contain a summary measure given by a prism below the single studies, but by a prism shown for each single study that shows the aggregated effect for the specific type of study (depending on the covariates of the meta-regression). If the heterogeneity test indicated that there was no substantial heterogeneity between studies, the Mantel-Haenszel method was fitted to calculate pooled estimates, $95 \%$ $\mathrm{Cls}$, and combined $p$ values. If substantial heterogeneity is indicated by $1250 \%$, the random-effects model will be performed using the DerSimonian and Laird method (DerSimonian 1986) and the rma function. The significance of the $p$-value represents the strength of evidence against the null hypothesis of no intervention effect. We will conduct Bayesian NMA using the Markov Chain Monte Carlo random effects model in 
Aggregate Data Drug Information System (ADDIS) version 1.16.8 (Drugis, Groningen, NL). We will network the translated outcomes within studies and specify the relations among the MD across studies, making different comparisons, as previously reported. We used $p<0.05$, and $95 \% \mathrm{Cl}$ beyond the null value to assess significance. We also calculated the inconsistency factor (IF) and $95 \% \mathrm{Cl}$ to evaluate the inconsistency of each closed loop, with an IF close to $\mathbf{0}$. In addition, the random effects variance and inconsistency variance were roughly equal, which is considered to be less inconsistent.

Subgroup analysis: The following subgroup analysis will be performed to assess the heterogeneity of the research: (1) Different types of non-pharmacological interventions. (2) Different intervention times for Windows. (3) Upper or lower limbs. (4)Different regions of the study In addition, if we detect any important and significant covariate contributing to the variation of the intervention effect by metaregression, subgroup analyses will also be conducted according to these covariates.

Sensitivity analysis: To confirm the robustness of our findings, a sensitivity analysis was conducted based on the different levels of bias of the included studies. To evaluate the internal validity of studies or treatment adequacy, we will subsequently remove studies with a high risk of bias, studies of unclear risk of bias, and studies of low risk of bias using the meta for package and leave out function.

Language: English.

Country(ies) involved: China.

Keywords: Stroke; Spasticity; Rehabilitation; Systematic review; network meta-analysis.

Dissemination plans: In addition to producing a report for the funders of this review, which will be made available free of charge on their website, a paper will be submitted to a leading journal in this field.
Contributions of each author:

Author 1 - Guanyu Hu - The author drafted the manuscript and assessed the risk of bias.

Email: 1241453233@qq.com

Author 2 - Hongshi Zhang - The author screened potential studies and extracted the data.

Email: 5503576@qq.com

Author 3 - Yufeng Wang - The author developed a searching strategy.

Email: wangchn@126.com

Author 4 - Deyu Cong - The author is the guarantor of the manuscript and drafted the manuscript.

Email: cong_de_yu@sina.com 